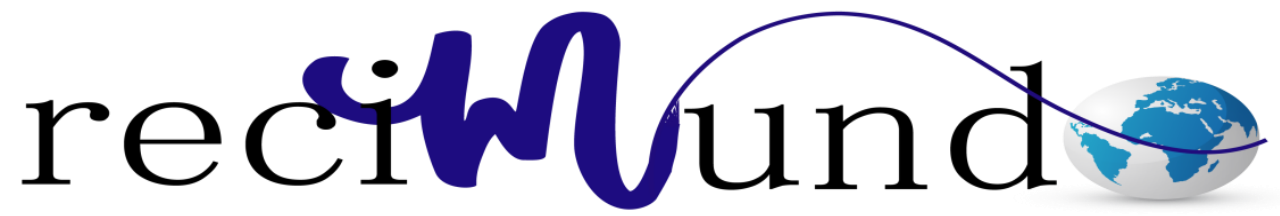

Revista Científica Mundo de la Investigación y el Conocimiento

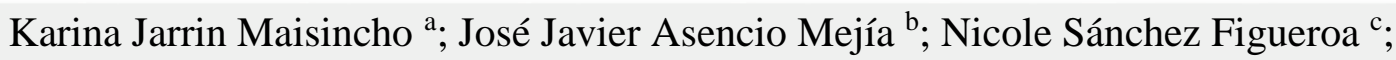
Wiliam Plua Marcillo ${ }^{\mathrm{d}}$

Modificación de parámetros en el síndrome metabólico en pacientes sometidos a gastrectomia vertical (manga gástrica)

Revista Científica Mundo de la Investigación y el Conocimiento. Vol. 2 núm., 1, febrero, ISSN: 2588-073X, 2018, pp. 202-223

DOI: $10.26820 /$ recimundo/2.1.2018.202-223

Editorial Saberes del Conocimiento

Recibido: 05/12/2017

Aceptado: 10/02/2018
a._drakarinajarrinmed@hotmail.com
b. josejavier_asencio@hotmail.com
c. Universidad de Especialidades Espíritu Santo; nicolesanchez@uees.edu.ec
d. wepm22@hotmail.com 


\section{Modificación de parámetros en el síndrome metabólico en pacientes sometidos \\ a gastrectomia vertical (manga gástrica)}

Vol. 2, núm. 1., (2018)

Karina Jarrin Maisincho; José Javier Asencio Mejía; Nicole Sánchez Figueroa; Wiliam Plua

Marcillo

\section{RESUMEN}

Introducción: la Gastrectomía Vertical Laparoscópica (GVL) o Manga Gástrica Laparoscópica ofrece resultados positivos en términos de reducción de peso, masa corporal, disminución de parámetros bioquímicos relacionados al síndrome metabólico con lo consiguiente disminución del riesgo cardiovascular y muerte. Objetivos: nuestro objetivo del estudio es determinar los efectos a un año posterior a la realización de la Gastrectomía Vertical Laparoscópica en los pacientes con síndrome metabólico y obesidad. Materiales y métodos: Nuestro estudio es de tipo retrospectivo, descriptivo en el cual incluyen 96 pacientes que fueron intervenidos de Gastrectomía Vertical Laparoscópica (LGS) entre el periodo de enero 2015 hasta diciembre 2017. Los datos obtenidos para el estudio fueron: IMC, peso, talla, Colesterol total, HDL, LDL, Triglicéridos y presión arterial. Además, se recopilaron las comorbilidades más frecuentes. Se realizó revisión de controles de hasta 1 año posterior a la cirugía. Resultados: Luego de un año de control postoperatorio se observó una disminución estadísticamente significativa (p: <0.0001) en relación a peso (de $111.41+2.26 \mathrm{~kg}$ a $77.14+1.65 \mathrm{~kg}$ ) e $\mathrm{IMC}(\mathrm{de} 43.08+0.8 \mathrm{~kg} / \mathrm{m} 2$ a 30.28 $+0.728 \mathrm{~kg} / \mathrm{m} 2$ ). En relación a presión arterial media al cabo de 1 año de control no demostraron diferencia significativa en términos de reducción $\mathrm{p}=0.39(91.13+1.68$ a $89.36+1.16)$. Se determinó una disminución estadísticamente significativa en los valores de glicemia plasmática en ayunas $(\mathrm{GPA}) \mathrm{p}=0.0130$ (de $103.57+6.28$ a $86.71+1.42)$. Los niveles de colesterol total, LDL y HDL no presentaron disminución al año de control postoperatorio. En cambio, los valores de triglicéridos si presentaron disminución importante al término de un año de control (152.16 + 7.42 a 101.18 + 5.51). Conclusión: La gastrectomía vertical laparoscópica ofrece un efecto positivo en términos de reducción de peso e imc, además de disminuir los niveles de glicemia, lo cual tendría mejoría en la evolución de los pacientes con obesidad y diabetes tipo 2. A pesar de presentar una disminución no significativa de la presión arterial, su reducción puede asociarse a una disminución en el riesgo cardiovascular asociado a obesidad.

Palabras clave: Gastrectomía Vertical Laparoscópica, Síndrome metabólico, obesidad, Manga gástrica.

Revista Científica Mundo de la Investigación y el Conocimiento. 2(1). pp. 202-223 


\section{Modificación de parámetros en el síndrome metabólico en pacientes sometidos a gastrectomia vertical (manga gástrica)}

Vol. 2, núm. 1., (2018)

Karina Jarrin Maisincho; José Javier Asencio Mejía; Nicole Sánchez Figueroa; Wiliam Plua Marcillo

\section{ABSTRACT}

Introduction: Laparoscopic Vertical Gastrectomy (GVL) or Laparoscopic Gastric Sleeve (LGS) offers positive results in terms of weight reduction, body mass, decrease in biochemical parameters related to the metabolic syndrome with consequent reduction in cardiovascular risk and death. Objectives: our objective of the study is to determine the effects a year after performing the Laparoscopic Vertical Gastrectomy in patients with metabolic syndrome and obesity. Materials and methods: Our study is a retrospective, descriptive study in which 96 patients were included who underwent a Laparoscopic Vertical Gastrectomy (LGS) between the period of January 2015 and December 2017. The data obtained for the study were: BMI, weight, size, total cholesterol, HDL, LDL, triglycerides and blood pressure. In addition, the most frequent comorbidities were collected. Controls were reviewed for up to 1 year after surgery. Results: After one year of postoperative control a statistically significant decrease was observed (p: $<0.0001$ ) in relation to weight (from $111.41+2.26 \mathrm{~kg}$ to $77.14+1.65 \mathrm{~kg}$ ) and BMI (from $43.08+0.8 \mathrm{~kg} / \mathrm{m} 2$ to $30.28+0.728 \mathrm{~kg} / \mathrm{m} 2$ ). In relation to mean arterial pressure after 1 year of control, they did not show a significant difference in terms of reduction $p=0.39(91.13+1.68$ to $89.36+1.16)$. A statistically significant decrease in fasting plasma glycaemia (GPA) values was determined $\mathrm{p}=0.0130$ (from $103.57+6.28$ to $86.71+1.42$ ). The levels of total cholesterol, LDL and HDL showed no decrease at one year of postoperative control. On the other hand, the values of triglycerides did show a significant decrease at the end of one year of control $(152.16+7.42$ to $101.18+5.51)$. Conclusion: Laparoscopic vertical gastrectomy offers a positive effect in terms of weight reduction and imc, in addition to lowering blood glucose levels, which would improve the evolution of patients with obesity and type 2 diabetes. Despite presenting a decrease not significant in blood pressure, its reduction can be associated with a decrease in cardiovascular risk associated with obesity.

Keywords: Laparoscopic Vertical Gastrectomy, Metabolic Syndrome, Obesity, Gastric Sleeve. 


\section{Modificación de parámetros en el síndrome metabólico en pacientes sometidos \\ a gastrectomia vertical (manga gástrica)}

Vol. 2, núm. 1., (2018)

Karina Jarrin Maisincho; José Javier Asencio Mejía; Nicole Sánchez Figueroa; Wiliam Plua

Marcillo

\section{Introducción.}

La obesidad es una entidad multifactorial que afecta a diferentes edades, representa globalmente entre un $2 \%$ a $10 \%$ de los costos de asistencia médica $(1,2)$. Su prevalencia ha aumentado durante el transcurso de los años. Reportes de datos estadísticos en EEUU demuestran que la prevalencia de obesidad en mujeres corresponde al 38\% y hombres el $36.9 \%$ hasta el 2013. En Sudamérica y Europa la prevalencia es más frecuente en mujeres entre un rango de 15 a $30 \%{ }^{1-4}$.

Alteraciones genéticas, cambios neurohormonales que regulan la ingesta de alimentos, aumento de los niveles de ansiedad, cambios en el estilo de vida, factores socioeconómicos y sedentarismo son algunas de las causas en la aparición de sobrepeso y obesidad. Los pacientes con obesidad en un gran porcentaje cursan con comorbilidades como hipertensión, diabetes mellitus, alteraciones osteomusculares que a su vez conllevan a un aumento de riesgo cardiovascular y muerte súbita ${ }^{5-7}$.

El paciente con obesidad presenta un gran desafío en cuanto a su tratamiento. Existen varias medidas terapéuticas, tanto clínicas como quirúrgicas. El manejo médico en los que destacan: cambios en el estilo de vida, terapia cognitiva-conductual y fármacos, en comparación al tratamiento quirúrgico no han demostrado su eficacia en la reducción de peso $(8,9)$. La mayoría de casos se relaciona su alto grado de ansiedad, pobre control y seguimiento de dietas de bajo índice glucémico y disminución de actividad deportiva. La cirugía para la obesidad en pocos meses logra disminuir más del $20 \%$ del peso corporal. 


\section{Modificación de parámetros en el síndrome metabólico en pacientes sometidos a gastrectomia vertical (manga gástrica)}

Vol. 2, núm. 1., (2018)

Karina Jarrin Maisincho; José Javier Asencio Mejía; Nicole Sánchez Figueroa; Wiliam Plua Marcillo

Las técnicas quirúrgicas tienen como efecto: restricción de alimentos o provocar malabsorción de nutrientes ${ }^{10-11} \quad$. En la actualidad la Manga gástrica y el Bypass son las técnicas quirúrgicas más frecuentes. Ambos procedimientos han demostrado buenos resultados para el tratamiento de la obesidad y disminuir las comorbilidades asociadas. Resaltando las técnicas malabsortivas para la remisión de diabetes tipo $2^{12}$.

El manejo del paciente con obesidad es multidisciplinario, requiere de un control estricto y sobre todo el deseo del paciente en acceder a tratamiento, teniendo en cuenta que los resultados óptimos son más visibles a largo plazo. En nuestro estudio a desarrollarse, se detallará los efectos asociados al tratamiento quirúrgico de Gastrectomía Vertical Laparoscópica en pacientes con obesidad y que fueron intervenidos en el Hospital Teodoro Maldonado Carbo durante el periodo de enero 2015 a diciembre 2016.

El objetivo de la presente investigación es determinar la disminución de los parámetros de síndrome metabólico en pacientes con obesidad mórbida intervenidos mediante Gastrectomía Vertical Laparoscópica (Manga Gástrica), en el Hospital Teodoro Maldonado Carbo 2015-2016.

\section{Metodología.}

Diseño de la investigación

Nuestro trabajo de titulación es un estudio de tipo retrospectivo y descriptivo realizado en el Hospital Teodoro Maldonado Carbo durante el periodo de tiempo comprendido entre Enero 2015 - Diciembre 2016, en el cual se realizó el procedimiento: Gastrectomía vertical laparoscópica (Manga Gástrica) entre los pacientes con diagnóstico de Obesidad (CIE 10 - E66). 


\section{Modificación de parámetros en el síndrome metabólico en pacientes sometidos \\ a gastrectomia vertical (manga gástrica)}

Vol. 2, núm. 1., (2018)

Karina Jarrin Maisincho; José Javier Asencio Mejía; Nicole Sánchez Figueroa; Wiliam Plua

Marcillo

Se realizó la recopilación de datos antropométricos, presión arterial y de exámenes de laboratorio relacionados a los parámetros del síndrome metabólico, que son los siguientes: peso, talla, índice de masa corporal, glucosa, colesterol total, HDLc, LDLc, triglicéridos y presión arterial. Los datos obtenidos abarcan desde su primera valoración preoperatoria, hasta el año posterior a su intervención quirúrgica. Además, se obtuvieron datos de comorbilidades asociadas previo a la cirugía.

Tipo de investigación

Estudio de tipo analítico no experimental, descriptivo y retrospectivo.

\section{Población}

La población está conformada por 139 pacientes que fueron sometidos a Gastrectomía vertical laparoscópica (Manga Gástrica) durante el periodo de enero 2015 - diciembre 2016.

Muestra

La muestra en nuestro estudio es a conveniencia, se encuentra integrada por un total de 96 pacientes. Estos pacientes cumplieron con sus evaluaciones preoperatorias multidisciplinarias, fueron sometidos a manga gástrica laparoscópica; además de contar con las valoraciones postoperatorias y resultados de exámenes de laboratorio completos durante el periodo enero 2015 - diciembre 2016.

Técnicas e instrumentos de la recolección de datos 


\section{Modificación de parámetros en el síndrome metabólico en pacientes sometidos a gastrectomia vertical (manga gástrica)}

Vol. 2, núm. 1., (2018)

Karina Jarrin Maisincho; José Javier Asencio Mejía; Nicole Sánchez Figueroa; Wiliam Plua Marcillo

Previa autorización y aprobación por el Jefe del Servicio de Cirugía General y Docencia, se obtuvieron los datos generales de los pacientes sometidos a manga gástrica y realizaron sus controles postoperatorios durante el periodo entre enero 2015 - diciembre 2016 en una base de datos de EXCEL por parte del servicio de Estadística.

Posteriormente se procedió a la revisión de las historias clínicas mediante el sistema informático de la institución AS400 y se procedió a la obtención de los datos individuales de los pacientes, los cuales fueron registrados en una base de datos de EXCEL 2010.

Técnica de análisis estadístico de la información

Para el análisis de los datos obtenidos, se utilizó el programa estadístico IBM SPSS previamente estos datos se introdujeron en una base creada en Microsoft Excel para Windows 10.

Se determinaron medidas de tendencia central (promedio, mediana, moda) y de dispersión (desviación estándar, error estándar) con el respectivo intervalo de confianza.

\section{Resultados.}

En el presente estudio se incluyeron en total 96 pacientes, de los cuales el 76.53\% (N= 75) correspondieron al sexo femenino y el $23.47 \%(\mathrm{~N}=23)$ al sexo masculino,

La edad promedio de los pacientes es de $40.9+1.93$ (37 - 44.7), con una mayor prevalencia entre 31 a 50 años de edad correspondiente al 30.21\% ( $\mathrm{N}=29)$. 


\section{Modificación de parámetros en el síndrome metabólico en pacientes sometidos}

a gastrectomia vertical (manga gástrica)

Vol. 2, núm. 1., (2018)

Karina Jarrin Maisincho; José Javier Asencio Mejía; Nicole Sánchez Figueroa; Wiliam Plua

Marcillo

\begin{tabular}{|lcc|}
\hline \multicolumn{3}{|c|}{ Distribución de los pacientes según el Sexo } \\
\hline Sexo & N & $\%$ \\
\hline Hombres & 23 & 23.47 \\
\hline Mujeres & 75 & 76.53 \\
\hline
\end{tabular}

Cuadro $N^{\circ}$ 1.- Distribución de los pacientes según el Sexo

\begin{tabular}{|lcc|}
\hline \multicolumn{2}{|c|}{ Distribución de los pacientes según la edad } \\
\hline \multicolumn{1}{|c|}{ Edad } & N & \% \\
\hline $\mathbf{2 0}$ - 30 años & 18 & 18,75 \\
\hline $\mathbf{3 1}$ - 40 años & 29 & 30,21 \\
\hline $\mathbf{4 1}$ - 50 años & 29 & 30,21 \\
\hline $\mathbf{5 1}$ - $\mathbf{6 0}$ años & 18 & 18,75 \\
\hline$>\mathbf{6 0}$ años & 2 & 2,08 \\
\hline
\end{tabular}

Cuadro $N^{\circ}$ 2.- Distribución de casos según la edad

\section{Evaluación de Medidas Antropométricas}

El peso prequirúrgico de los pacientes fue de $111.41+2.26(106.89$ - 115.92) y al año de la intervención quirúrgica presenta 77.14 + 1.65 (73.83 - 80.45). En relación al IMC el valor prequirúrgico fue de $43.08+0.8(41.45-44.67)$ y al año de realizarse manga gástrica presenta $30.28+0.72(28.84-31.71)$ resultando en una diferencia de medias de $12.80+0.50(11.80-$ 13.80) $\mathrm{p}=<0.0001 \quad \mathrm{t}: 25.44 \mathrm{df}: 64 \mathrm{p}:<0.0001$. 
Modificación de parámetros en el síndrome metabólico en pacientes sometidos a gastrectomia vertical (manga gástrica)

Vol. 2, núm. 1., (2018)

Karina Jarrin Maisincho; José Javier Asencio Mejía; Nicole Sánchez Figueroa; Wiliam Plua Marcillo

\begin{tabular}{|lcc|}
\hline \multicolumn{3}{|c|}{ Distribución de los pacientes según datos antropométricos. } \\
\hline \multicolumn{3}{|c|}{ Peso } \\
\hline IMC \\
\hline $\begin{array}{c}111.41 \pm 2.26(106.89- \\
115.92)\end{array}$ & $43.08 \pm 0.8(41.45-44.67)$ \\
\hline 1er mes (PO) & $\begin{array}{c}96.73 \pm 1.76(93.22- \\
100.24)\end{array}$ & $37.94 \pm 0.65(36.65-39.23)$ \\
\hline 6 meses (PO) & $83.64 \pm 1.93(79.77-87.51)$ & $32.82 \pm 0.68(31.46-34.18)$ \\
\hline $\mathbf{1 2}$ meses (PO) & $77.14 \pm 1.65(73.83-80.45)$ & $30.28 \pm 0.72(28.84-31.71)$ \\
\hline
\end{tabular}

Cuadro $N^{\circ}$ 3.- Distribución de los pacientes según los datos antropométricos.

$\mathrm{PO}=$ postoperatorio

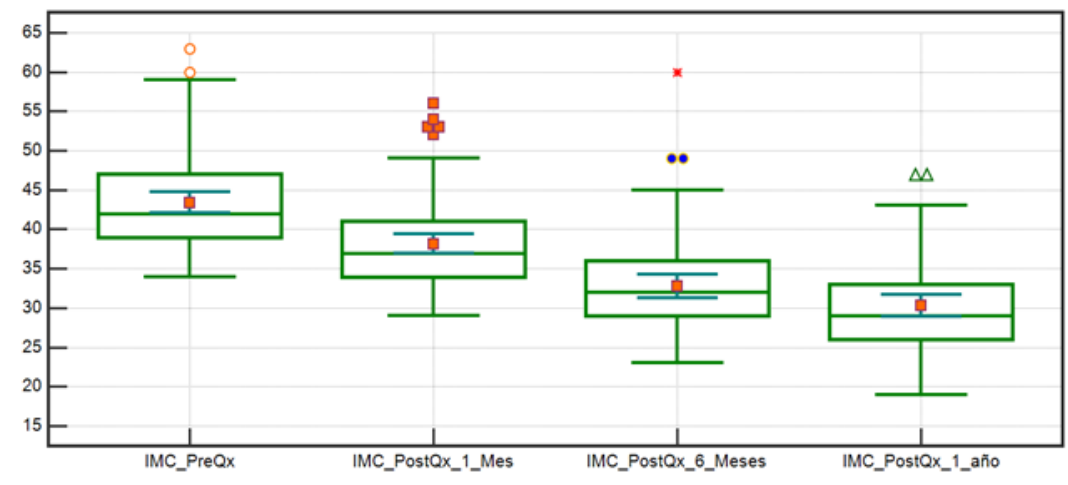

Figura $N^{\circ}$ 1.- Valoración del índice de masa corporal mediante diagrama de cajas.

Evaluación de la Presión Arterial. 


\section{Modificación de parámetros en el síndrome metabólico en pacientes sometidos}

a gastrectomia vertical (manga gástrica)

Vol. 2, núm. 1., (2018)

Karina Jarrin Maisincho; José Javier Asencio Mejía; Nicole Sánchez Figueroa; Wiliam Plua

Marcillo

Al evaluar los valores de la Presión arterial media se encontró un valor prequirúrgico de $91.13+1.68(87.77-94.49)$ y al año de la intervención fue de $89.36+1.16(87.02-91.69)$, resultando en una diferencia de medias de $1.77+2.05(2.35-5.89) \mathrm{t}=0.86 \mathrm{df}: 52 \mathrm{p}=0.39$.

\section{Valores de Presión Arterial Media}

Preoperatorio $\quad 91.13 \pm 1.68(87.77-94.49)$

1er mes (PO) $\quad 89.72 \pm 1.073(87.57-91.86)$

6 meses (PO) $\quad 89.21 \pm 1.04(87.14-91.28)$

12 meses (PO) de $89.36 \pm 1.16(87.02-91.69)$

\section{Cuadro $N^{\circ}$ 4.- Valoración cronológica de la Presión arterial media.}

$\mathrm{PO}=$ postoperatorio.

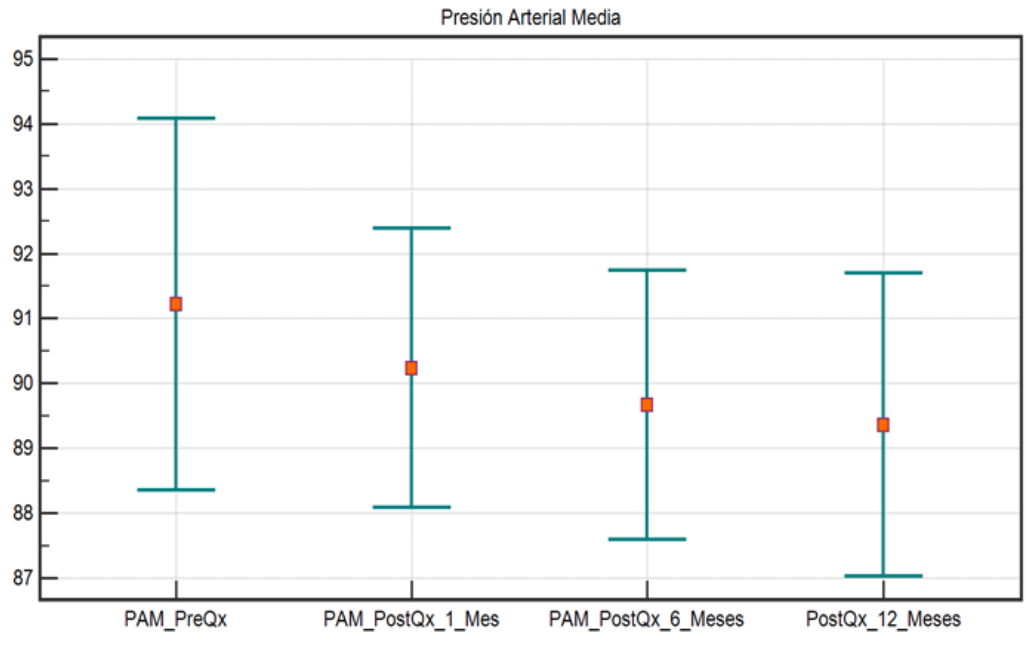

Figura $N^{\circ}$ 2.- Valoración cronológica de la presión arterial 


\section{Modificación de parámetros en el síndrome metabólico en pacientes sometidos}

a gastrectomia vertical (manga gástrica)

Vol. 2, núm. 1., (2018)

Karina Jarrin Maisincho; José Javier Asencio Mejía; Nicole Sánchez Figueroa; Wiliam Plua Marcillo

\section{Valores de Hemoglobina y Hematocrito}

El valor prequirúrgico de Hemoglobina fue de $13.46+0.15(13.16-13.75)$ y al año de realizarse la manga gástrica los pacientes presentaron un valor promedio de hemoglobina de $12.77+0.19(13.14-12.40)$ con una diferencia de medias de $0.69+0.25(0.19-1.18)$ t: 2.77 df:52 $\mathrm{p}=0.007$.

\section{Valores de Hemoglobina y Hematocrito}

\begin{tabular}{|lcc|} 
Control & Hemoglobina & Hematocrito \\
\hline Preoperatorio & $13.46 \pm 0.15(13.16-$ & $40,85 \pm 0.30(35.17-$ \\
& $13.75)$ & $46.52)$ \\
\hline 1er mes (PO) & $90.35 \pm 1.99(86.38-$ & $39.21 \pm 0.32(33.52-$ \\
& $94.32)$ & $44.89)$ \\
\hline 6 meses (PO) & $86.41 \pm 1.39(83.63-$ & $39.44 \pm 0.41(32.83-$ \\
& $89.19)$ & $46,05)$ \\
\hline 12 meses (PO) & $12.77 \pm 0.19(13.14-$ & $38.73 \pm 0.55(30.62-$ \\
& $12.40)$ & $46.83)$ \\
\hline
\end{tabular}

Cuadro $N^{\circ}$ 5.- Niveles de Hemoglobina y Hematócrito en pre y postoperatorio.

$\mathrm{PO}=$ postoperatorio 


\section{Modificación de parámetros en el síndrome metabólico en pacientes sometidos}

a gastrectomia vertical (manga gástrica)

Vol. 2, núm. 1., (2018)

Karina Jarrin Maisincho; José Javier Asencio Mejía; Nicole Sánchez Figueroa; Wiliam Plua

Marcillo

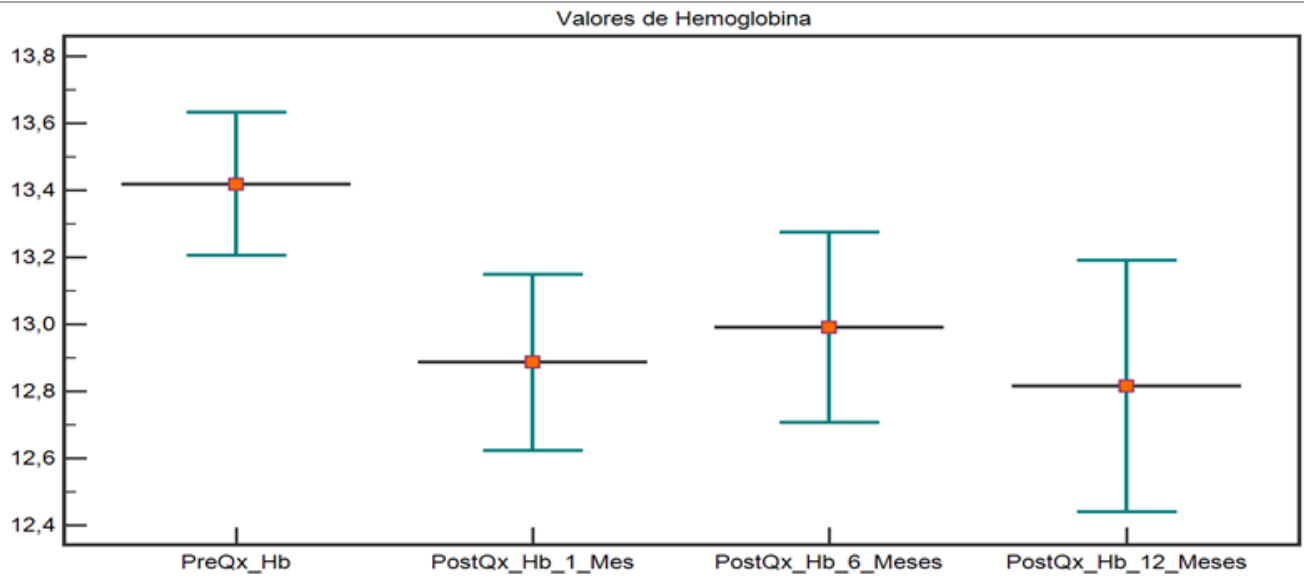

Figura $N^{\circ}$ 3.- Valoración cronológica de Hemoglobina

EVALUACION DE DISLIPIDEMIA

\begin{tabular}{|c|c|c|c|c|}
\hline & Preoperatorio & $1 \mathrm{Mes}(\mathrm{PO})$ & 6 Meses (PO) & $\begin{array}{c}12 \text { Meses } \\
\text { (PO) }\end{array}$ \\
\hline $\begin{array}{l}\text { Colesterol } \\
\text { Total }\end{array}$ & $\begin{array}{l}189.62 \pm 3.64 \\
182.33- \\
196.90)\end{array}$ & $\begin{array}{l}173.71 \pm \\
7.13(159.46 \\
-187.96)\end{array}$ & $\begin{array}{l}190.72 \pm 5.26 \\
(180.19- \\
201.25)\end{array}$ & $\begin{array}{l}191.74 \pm \\
6.20 \\
(179.33- \\
204.14)\end{array}$ \\
\hline LDL & $\begin{array}{l}125.79 \pm 3.12 \\
(119.56-132.03)\end{array}$ & $\begin{array}{l}116.65 \pm \\
4.63(107,39 \\
-125,90)\end{array}$ & $\begin{array}{l}113.28 \pm 5.88 \\
(101.51- \\
125.04)\end{array}$ & $\begin{array}{l}121.38 \pm \\
6.56 \\
(108.27- \\
134.49)\end{array}$ \\
\hline HDL & $\begin{array}{l}42.26 \pm 1.03 \\
(40.20-44.32)\end{array}$ & $\begin{array}{l}40.89 \pm 1.48 \\
(37.93- \\
43.86)\end{array}$ & $\begin{array}{l}55.05 \pm 3.60 \\
(47.85- \\
62.24)\end{array}$ & $\begin{array}{l}68.86 \pm \\
11.01 \\
(46.83- \\
90.89)\end{array}$ \\
\hline Triglicéridos & & $111.84 \pm$ & $97.67 \pm 5.08$ & $101.18 \pm$ \\
\hline
\end{tabular}




\section{Modificación de parámetros en el síndrome metabólico en pacientes sometidos a gastrectomia vertical (manga gástrica)}

Vol. 2, núm. 1., (2018)

Karina Jarrin Maisincho; José Javier Asencio Mejía; Nicole Sánchez Figueroa; Wiliam Plua Marcillo

\begin{tabular}{l|l|l|l|}
$152.16 \pm 7.42$ & $6.33(99.18-$ & $(87.51-$ & $5.51(90.17$ \\
$(137.31-167)$ & $124.50)$ & $107.82)$ & $-112.20)$
\end{tabular}

\section{Cuadro $N^{\circ}$ 6.- Valores de perfil lipídico en pacientes pre y postoperatorio.}

$\mathrm{PO}=$ postoperatorio

Los valores de colesterol total disminuyen al mes de la cirugía $(173.71+7.13$ (159.46 187.96)), pero en comparación al año, los valores se encuentran por encima del valor de inicio del estudio (191.74 + 6.20 (179.33 - 204.14)). Resultados similares se observan en LDL y HDL. Únicamente los triglicéridos disminuyeron al año de la cirugía en relación a sus valores al inicio del estudio $(101.18+5.51(90.17-112.20))$.

Glicemia Plasmática en Ayunas

Los valores pre quirúrgico de Glicemia Plasmática en ayunas corresponde a 103.57 + 6.28 (90.94 - 116.20) en comparación con los datos reportados al año de la intervención quirúrgica siendo $86.71+1.42(83.84-89.57)$, correspondiente a una diferencia de medias de $16.86+6.53(3.72-30.00) \mathrm{p}=0.0130$.

\begin{tabular}{|l|l|}
\hline \multicolumn{2}{|c|}{ Niveles de Glicemia Plasmática en Ayunas } \\
\hline GPA & $103.57 \pm 6.28(90.94-116.20)$ \\
\hline Prequirúrgico & $90.35 \pm 1.99(86.38-94.32)$ \\
\hline 1 Mes & $86.41 \pm 1.39(83.63-89.19)$ \\
\hline 6 Meses & $86.71 \pm 1.42(83.84-89.57)$ \\
\hline 12 Meses &
\end{tabular}

Cuadro $N^{\circ}$ 7.- Cambios postoperatorios en los niveles de glicemia plasmática en ayunas.

Revista Científica Mundo de la Investigación y el Conocimiento. 2(1). pp. 202-223 


\section{Modificación de parámetros en el síndrome metabólico en pacientes sometidos \\ a gastrectomia vertical (manga gástrica)}

Vol. 2, núm. 1., (2018)

Karina Jarrin Maisincho; José Javier Asencio Mejía; Nicole Sánchez Figueroa; Wiliam Plua

Marcillo

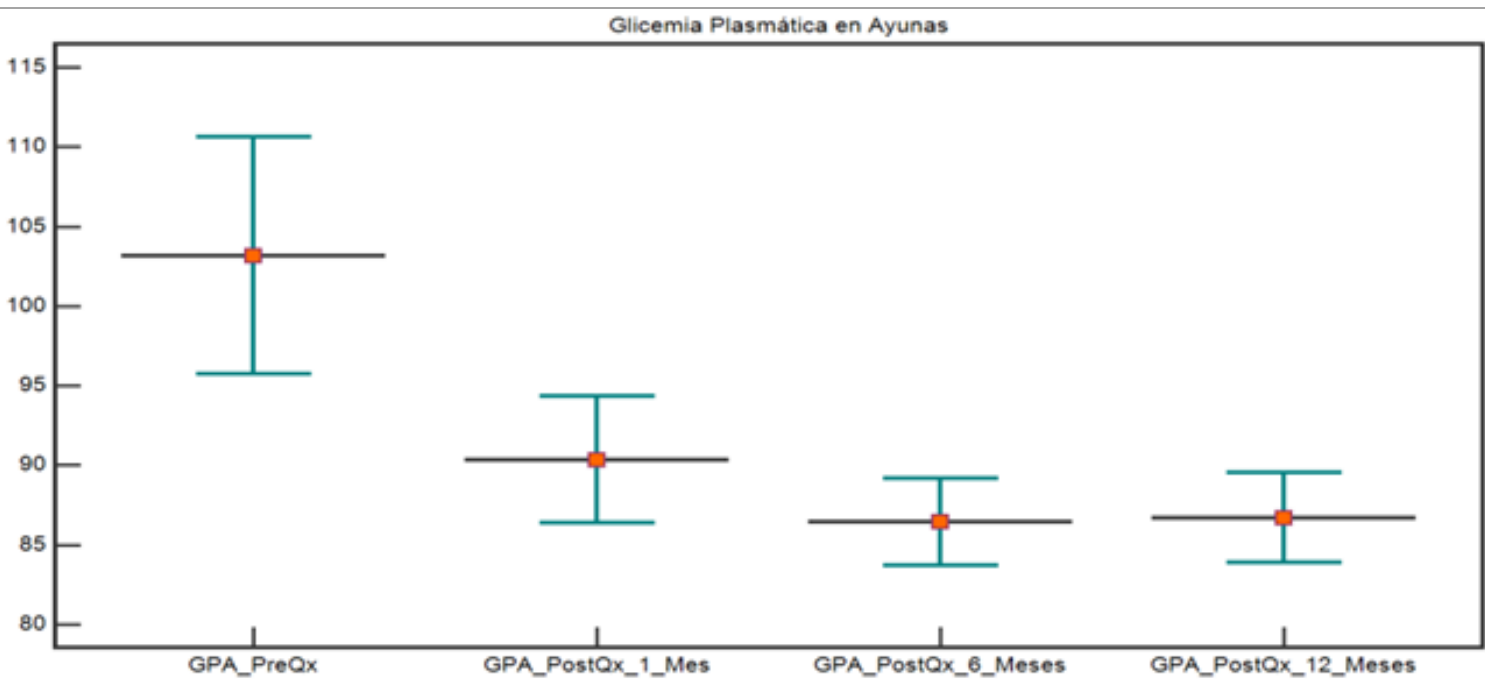

Figura $N^{\circ}$ 4.- Valoración cronológica de niveles de glicemia plasmática en ayunas

\section{Valoración de Comorbilidades}

De acuerdo a la nueva definición de la IDF (International Diabetes Federation), para que una persona tenga síndrome metabólico debe tener: Obesidad central (definido como circunferencia de cintura $>/=94 \mathrm{~cm}$ para hombres caucásicos y $>/=80 \mathrm{~cm}$ para mujeres caucásicas, con valores étnicos específicos para otros grupos), Más dos de los siguientes 4 factores: Nivel de triglicéridos (TG) elevados: $>/=150 \mathrm{mg} / \mathrm{dL}(1,7 \mathrm{mmol} / \mathrm{L})$, o tratamiento específico para esta anormalidad lipídica, Colesterol HDL reducido: $<40 \mathrm{mg} / \mathrm{dL}(1,03 \mathrm{mmol} / \mathrm{L})$ en hombres y $<50 \mathrm{mg} / \mathrm{dL}(1,29 \mathrm{mmol} / \mathrm{L})$ en mujeres, o tratamiento específico para esta anormalidad lipídica, Tensión arterial (TA) elevada: TA sistólica $>/=130$ o TA diastólica $>/=$ $85 \mathrm{~mm} \mathrm{Hg}$, o tratamiento de hipertensión previamente diagnosticada, Glucosa plasmática en ayunas elevada $>/=100 \mathrm{mg} / \mathrm{dl}(5,6 \mathrm{mmol} / \mathrm{L})$, o diabetes tipo 2 previamente diagnosticada. Si la 


\section{Modificación de parámetros en el síndrome metabólico en pacientes sometidos a gastrectomia vertical (manga gástrica)}

Vol. 2, núm. 1., (2018)

Karina Jarrin Maisincho; José Javier Asencio Mejía; Nicole Sánchez Figueroa; Wiliam Plua Marcillo

glucosa en ayunas es $>5,6 \mathrm{mmol} / \mathrm{L}$ o $100 \mathrm{mg} / \mathrm{dl}$, la prueba de tolerancia oral a la glucosa (PTOG) es fuertemente recomendada pero no es necesaria para definir la presencia del síndrome.

Los pacientes que cumplieron criterios de Síndrome Metabólico correspondieron al 84\% $(\mathrm{N}=81)$ de la muestra, y posteriormente al año de ser valorados el $61 \%(\mathrm{~N}=59)$ de los pacientes incluidos en el estudio presentaron Síndrome metabólico.

En el presente estudio se encontró que las comorbilidades más frecuentes fueron: Hipertensión arterial (47\%), Diabetes mellitus tipo 2 (54\%) y Dislipidemia (21\%). Al año posterior a la intervención quirúrgica el porcentaje de pacientes en la cual persistieron estas comorbilidades fue de: 31\% (HTA), 48\% (DM2) y 18\% (Dislipidemia).

\begin{tabular}{|l|c|c|}
\hline \multicolumn{3}{|c|}{ Tipos de comorbilidades más frecuentes } \\
\hline Comorbilidades & Preoperatorio & 12 Meses \\
\hline HTA & $41,00 \%(\mathrm{~N}=39)$ & $34,00 \%(\mathrm{~N}=33)$ \\
\hline DM2 & $54,00 \%(\mathrm{~N}=52)$ & $25,00 \%(\mathrm{~N}=24)$ \\
\hline Dislipidemia & $21,00 \%(\mathrm{~N}=20)$ & $16,00 \%(\mathrm{~N}=15)$ \\
\hline
\end{tabular}

Cuadro $N^{\circ}$ 8.- Valoración de las comorbilidades más frecuentes y el porcentaje de disminución durante el estudio 


\section{Modificación de parámetros en el síndrome metabólico en pacientes sometidos \\ a gastrectomia vertical (manga gástrica)}

Vol. 2, núm. 1., (2018)

Karina Jarrin Maisincho; José Javier Asencio Mejía; Nicole Sánchez Figueroa; Wiliam Plua

Marcillo

\section{Discusión.}

La obesidad ha alcanzado proporciones epidémicas a nivel mundial, y cada año mueren, como mínimo, 2,8 millones de personas a causa de la obesidad o sobrepeso. Aunque anteriormente se consideraba un problema confinado a los países de altos ingresos, en la actualidad la obesidad también es prevalente en los países de ingresos bajos y medianos ${ }^{13}$.

Un IMC elevado es un importante factor de riesgo de enfermedades no transmisibles, como: las enfermedades cardiovasculares (principalmente las cardiopatías y los accidentes cerebrovasculares), que fueron la principal causa de muertes en 2012; la diabetes; los trastornos del aparato locomotor (en especial la osteoartritis), y algunos cánceres (endometrio, mama, ovarios, próstata, hígado, vesícula biliar, riñones y colon). El riesgo de contraer estas enfermedades no transmisibles crece con el aumento del $\mathrm{IMC}^{14}$.

El incremento del Índice de Masa corporal, además tiene mucha influencia sobre la calidad de vida, ya que pueden verse afectados los componentes físico, emocional, social y psicológico de los pacientes. Debido a los datos epidemiológicos presentados, es importante prestar atención a las consecuencias que conlleva el sobrepeso y la obesidad sobre nuestra salud además de adoptar todas las medidas necesarias para reducir la prevalencia de obesidad en nuestra población.

Las medidas primarias a tomar en cuenta corresponden a los cambios en el estilo de vida, es decir, alimentación saludable, dieta, ejercicio, los cuales en la mayoría de situaciones no logran ser llevadas a cabalidad por falta de tiempo, disciplina, motivación, interés, siendo en la 


\section{Modificación de parámetros en el síndrome metabólico en pacientes sometidos a gastrectomia vertical (manga gástrica)}

Vol. 2, núm. 1., (2018)

Karina Jarrin Maisincho; José Javier Asencio Mejía; Nicole Sánchez Figueroa; Wiliam Plua Marcillo

mayoría de las ocasiones perdidas temporales de peso. Los beneficios que ofrece la Manga gástrica tienen como logros alcanzados una reducción del exceso de peso, del índice de masa corporal, de las comorbilidades asociadas, sin afectar la calidad de vida de los pacientes.

En el presente estudio, realizado en la ciudad de Guayaquil en el hospital Teodoro Maldonado Carbo durante el periodo de tiempo comprendido entre enero 2015 a Diciembre 2016 reunieron todos los criterios de inclusión 96 pacientes los cuales fueron intervenidos por Manga gástrica. De los pacientes incluidos en el estudio se encontró que la mayor prevalencia corresponde al sexo femenino siendo el $77 \%$ de la población incluida en el estudio. Además la edad promedio de los pacientes fue de $40.9 \pm 1.93(37-44.7)$

Todos los pacientes incluidos presentaron un Índice de Masa Corporal > $30 \mathrm{Kg} / \mathrm{m} 2$ los mismos que al ser sometidos a intervención quirúrgica por Manga Gástrica presentaron una reducción del IMC de $12.80 \pm 0.50(11.80$ - 13.80) Kg al año de ser intervenidos. Así pues, el peso pre quirúrgico de los pacientes fue de $111.41 \pm 2.26(106.89-115.92)$ y al año de la intervención quirúrgica presenta $77.14 \pm 1.65$ (73.83 - 80.45). En relación al IMC el valor pre quirúrgico fue de $43.08 \pm 0.8(41.45$ - 44.67) y al año de realizarse manga gástrica presenta $30.28 \pm 0.72(28.84-31.71)$. Datos similares fueron encontrados en el estudio Monserrato y cols. realizado en el Hospital Teodoro Maldonado Carbo en la ciudad de Guayaquil, donde del total de la muestra estudiada (155 pacientes), se observó un descenso del promedio global del peso en el transcurso de la evolución postquirúrgico, se inició con un peso pre-quirúrgico de 120,8 kg y culminó con un peso de $97,4 \mathrm{~kg}$ a los 9 meses de post-quirúrgico ${ }^{15}$. De manera similar el estudio publicado por Hady Razak y col. demostró también una disminución 


\section{Modificación de parámetros en el síndrome metabólico en pacientes sometidos \\ a gastrectomia vertical (manga gástrica)}

Vol. 2, núm. 1., (2018)

Karina Jarrin Maisincho; José Javier Asencio Mejía; Nicole Sánchez Figueroa; Wiliam Plua

Marcillo

significativa del peso e IMC de $53.18 \pm 7.5 \mathrm{~kg} / \mathrm{m} 2$ a $31.4 \pm 3.75 \mathrm{~kg} / \mathrm{m} 2$ al año de realizada la cirugía ${ }^{16}$.

Sin embargo estudios han reportado que existe reganancia de peso a partir de los 2 años de ser intervenidos los pacientes. Se ha reportado desde 20 a $87 \%$ de pacientes con reganancia de peso en diversas series. El aumento de peso ocurre más frecuentemente entre 3 y 6 años después de la cirugía. La reganancia de un $25 \%$ del peso perdido previamente por efecto de la cirugía bariátrica ha sido considerada por algunos autores como un criterio suficiente para realizar cirugía de revisión. En otros estudios se ha considerado que la reganancia de peso ha favorecido la reaparición de comorbilidades como la Diabetes ${ }^{17}$.

En nuestro estudio al evaluar las comorbilidades se encontró en los datos prequirúrgicos que el $41 \%$ de los pacientes incluidos presentaron antecedentes de Hipertensión, el 54\% Diabetes Mellitus tipo 2 y el 21\% Dislipidemia, sin embargo hay que tener en cuenta que muchos pacientes incluidos en el estudio desconocía su estado de salud real, y manifestaban no presentar antecedentes patológicos personales, sin embargo en las mediciones de las variables se encontraban alteraciones de las mismas, razón por la que, es muy probable que estos datos sean incluso mayores de los reportados.

Al año de ser intervenidos los pacientes, se evaluó la afectación sobre las comorbilidades, así pues del $41 \%$ de pacientes con hipertensión arterial, al año mantenían este antecedente el 34\%, En relación a la Diabetes Mellitus se encontró un efecto más favorable, del 54\% de pacientes reportados en el prequirúrgico, presentaron resolución de la enfermedad más de la mitad de los pacientes al año de ser intervenidos. Sin embargo, existen otros estudios con datos 


\section{Modificación de parámetros en el síndrome metabólico en pacientes sometidos a gastrectomia vertical (manga gástrica)}

Vol. 2, núm. 1., (2018)

Karina Jarrin Maisincho; José Javier Asencio Mejía; Nicole Sánchez Figueroa; Wiliam Plua Marcillo

que presentan mayor impacto sobre las comorbilidades, así pues en el estudio publicado por el Dr. García Ruiz, se observó que la resolución de las comorbilidades asociadas a la obesidad, en promedio, la diabetes remitió en un $55 \%$ y la hipertensión en un $70 \%{ }^{18}$.

Se han realizado varios estudios relacionados con la cirugía bariátrica dirigidos a demostrar sus efectos a largo plazo. Un estudio que dio seguimiento a pacientes durante ocho años (2002-2010) fue el realizado por De la Cruz y su grupo, del que destaca el seguimiento a 1,603 adultos con IMC mayor de 35 con más de un factor de comorbilidad asociada o IMC mayor de 40 sin morbilidad a quienes posteriormente se realizó bypass gástrico o se les colocó una banda gástrica. De los 1,603 pacientes, 377 ya tenían el diagnóstico de diabetes mellitus tipo 2; 276 de prediabetes y 843 con concentraciones normales de glucosa. En los resultados del estudio la disminución de peso fue evidente, El descenso importante del peso logró obtener cambios en la glucosa, el grupo de los no diagnosticados con diabetes mellitus tipo 2 tuvo mejor descenso de glucosa (70.20 mg/dL 43\%), seguido de los pacientes del grupo con diagnóstico de diabetes mellitus tipo $2(49.09 \mathrm{mg} / \mathrm{dL} 33 \%)^{19}$.

En la valoración de los efectos sobre la dislipidemia, se encontró una mejoría mínima de la misma, estos datos son similares a los encontrados en otros estudios, donde la mejoría de la dislipidemia fue solo del $35 \%$ en pacientes intervenidos por Manga Gástrica, sin embargo la resolución en el 100\% de los valores de colesterol y triglicéridos se encontró a los 4 años de seguimiento $^{20}$.

Dentro de las limitaciones del presente estudio se encuentran: el tamaño muestral, la pérdida del seguimiento de los pacientes ya que muchos no acuden a las consultas de cirugía en 


\section{Modificación de parámetros en el síndrome metabólico en pacientes sometidos \\ a gastrectomia vertical (manga gástrica)}

Vol. 2, núm. 1., (2018)

Karina Jarrin Maisincho; José Javier Asencio Mejía; Nicole Sánchez Figueroa; Wiliam Plua

Marcillo

las fechas programadas, además que a partir del año de la intervención quirúrgica, la mayoría no acude más a controles ni valoración nutricional.

\section{Conclusiones.}

La gastrectomía vertical es un procedimiento que brinda buenos resultados en relación a la disminución de los niveles de glicemias plasmáticas en ayunas en un año de control. (103.57 $\mathrm{mg} / \mathrm{dl}+6.28 \mathrm{a} 86.71+1.42) \mathrm{p}=0.0130$.

En relación a la disminución de Peso e IMC la gastrectomía vertical permite una excelente reducción a un año de control. Peso (de $111.41 \mathrm{Kg}+2.26$ a 77.14Kg + 1.65) e IMC (de $30.28 \mathrm{Kg} / \mathrm{m} 2+0.72$ a $12.80 \mathrm{Kg} / \mathrm{m} 2+0.50) \mathrm{p}=<0.0001$.

La gastrectomía vertical produce cambios mínimos en los niveles de presión arterial a un año de control. PAM (de $91.13 \mathrm{mmHg}+1.68$ a $89.36 \mathrm{mmHg}+1.16) \mathrm{p}=0.39$.

La gastrectomía vertical produce cambios a un año en el perfil lipídico. Identificándose mayor reducción en los valores de Triglicéridos (de $152.16 \mathrm{mg} / \mathrm{dl}+7.42$ a $101.18 \mathrm{mg} / \mathrm{dl}+5.51)$.

\section{Bibliografía.}

1. Bray GA. Obesity. In Elsevier, editor. Sleisenger and Fordtran's Gastrointestinal and Liver Disease, Tenth Edition. Philadelphia: Saunders; 2016. p. 102-118.

2. Maira Bes-Rastrollo FJBGyJAMH. Epidemiología y prevención de la obesidad. In Elsevier, editor. Conceptos de salud pública y estrategias preventivas. 1st ed. España: Elsevier; 2013. p. 133-142.

3. Atalah E. Epidemiología de la obesidad en chile. Revista Médica Clínica Las Condes. 2012 
Modificación de parámetros en el síndrome metabólico en pacientes sometidos a gastrectomia vertical (manga gástrica)

Vol. 2, núm. 1., (2018)

Karina Jarrin Maisincho; José Javier Asencio Mejía; Nicole Sánchez Figueroa; Wiliam Plua Marcillo

Marzo; 23(2): p. 117-123.

4. Bhupathiraju H, Frank B. Epidemiology of Obesity and Diabetes and Their Cardiovascular. HHS. 2016 Mayo; 118(11).

5. Fatima Cody Stanford MMM. Dynamed Plus. [Online].; 2016 [cited 2016 Diciembre 7. Available from: https://www.dynamed.com/topics/dmp AN T115009/Obesity-inadults\#Epidemiology.

6. Hutcher NE. Incidencia, prevalencia y demografía de la obesidad. In Elsevier, editor. Cirugia de la Obesidad. España: Elsevier; 2009. p. 10-17.

7. Hanna-Maaria L, Bouchard C. Etiología de la obesidad. In Elsevier, editor. Cirugia de la Obesidad. España: Elsevier; 2009. p. 1828.

8. Kadouh HC, Acosta A. Current paradigms in the etiology of obesity. Techniques in Gastrointestinal Endoscopy. 2017 Enero; 19(1): p. 2-11.

9. Kushner RF. Valoración y tratamiento. In Hill MG, editor. Harrison Principios de Medicina Interna. 19th ed. Estados Unidos: Mc Graw Hill; 2015. p. 2392-2398.

10. M.D. CLB. Obesity. In FERRI FF, editor. Ferri's Clinical Advisor. Rhode Island: Elsevier; 2017. p. 906-909.

11. Michael A. Cowley WAByRVC. Obesity: The Problem and Its Management. In Saunders , editor. Endocrinology: Adult and Pediatric, Seventh Edition. Philadelphia: Elsevier; 2016. p. 468-473.

12. Jensen MD. Michael D. Jensen. In Elsevier, editor. Goldman-Cecil. Tratado de medicina

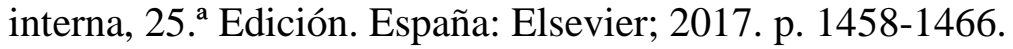

13. OMS. Organizaciòn Mundial de la Salud. [Online].; 2017 [cited 2017 Octubre 12. Available from: http://www.who.int/features/factfiles/obesity/es/.

14. WHO. World Health Organization. [Online].; 2017 [cited 2017 Octubre 12. Available from: http://www.who.int/mediacentre/factsheets/fs311/es/.

15. Juan Monserrate Cervantes WBIPPSWPMBMGCVCJM. MANGA GÁSTRICA EN PACIENTES CON OBESIDAD MÓRBIDA EN EL HOSPITAL DR. TEODORO MALDONADO CARBO GUAYAQUIL-ECUADOR ENERO DE 2011 A DICIEMBRE DE 2012. CIENCIAS PEDAGÒGICAS E INNOVACIÒN. 2016 Noviembre; 4(2). 


\section{Modificación de parámetros en el síndrome metabólico en pacientes sometidos}

a gastrectomia vertical (manga gástrica)

Vol. 2, núm. 1., (2018)

Karina Jarrin Maisincho; José Javier Asencio Mejía; Nicole Sánchez Figueroa; Wiliam Plua

Marcillo

16. Hady HR. The Influence of Laparoscopic Sleeve Gastrectomy on Metabolic Syndrome Parameters in Obese Patients in Own Material. Obesity Surgery - Springer. 2011 Octubre; 22(1).

17. V DKP. Reganancia de peso después de la cirugía bariátrica. Revista chilena de cirugía.. 2012 Febrero; 64(1).

18. Ruiz G. Gastrectomía en manga: Resultados a mediano y largo plazo. Cirujano General medigraphic. 2012 Enero; 34(1).

19. Solís-Ayala E, Carrillo-Ocampo L, Canché-Arenas A, Cortázar-Benítez L, Cabrera-Jardines R, Rodríguez-Weber F. Cirugía bariátrica: resultados metabólicos y complicaciones. Medicina Interna de México. 2013 Mayo; 29(5).

20. González LMP. Manga gástrica laparoscópica. Medigraphic. 2014 Noviembre; 57(6). 